\title{
Rapid tumor progression in a patient with HPV type 16 associated anal squamous cell carcinoma suffering from long-standing Crohn's disease: A case report
}

\author{
Fischer $\mathrm{AK}^{1,{ }^{*}}$, Krause $\mathrm{T}^{2}$, Steinbrück $\mathrm{H}^{2}$, Schildhaus $\mathrm{HU}^{3}$, Hoppenau $\mathrm{C}^{3}$, Hesterberg $\mathrm{R}^{4}$ and Rüschoff $\mathrm{J}^{1}$ \\ 1 Institute of Pathology Nordhessen, Germaniastraße 7, 34119 Kassel, Germany \\ 2 Gastroenterology Practice, Opernstraße 9, 34117 Kassel, Germany \\ ${ }^{3}$ Institute of Pathology, University Hospital Göttingen, Robert-Koch-Straße 40, 37075 Göttingen, Germany \\ ${ }^{4}$ Rotes Kreuz Krankenhaus, Chirurgische Klinik, Hansteinstraße 29, 34121, Kassel, Germany
}

\begin{abstract}
Background and aim: Squamous cell carcinoma (SCC) is the most common cancer of the anal region, typically associated with high-risk (hr) HPV infection. Furthermore, there is evidence that Crohn's disease predisposes to adenocarcinoma in patients with perianal disease. Materials and methods: A 57-year old patient presenting with long history of Crohn's disease since the age of mid-twenties, went through several surgeries including ileocolectomy and anal fistula resection, combined with immunosuppressive therapy additionally periodically since 2008. One year before death (in 2015) a painful fistula was diagnosed with extensive high grade anal intraepithelial neoplasia (AIN$\mathrm{HG}$ ) and evidence of invasive growth as non-keratinizing SCC. Tissue samples from several previous and current resection specimens were re-evaluated and extensively investigated for Crohn's type inflammation, dysplasia and HPV both by immunohistochemistry (p16/Ki67) and molecular subtyping of HPV. Results: AIN-HG and invasive anal squamous cell carcinoma turned out to be strongly positive for p16/ Ki67 staining and molecular analysis disclosed a HPV-16 subtype. In contrast, HPV-analysis was negative in all available previous tissue samples including one anal fistula resected five years before (in 2009) which was lined by non-keratinized squamous epithelium without any evidence of dysplasia. Thus, the patient was diagnosed as Crohn's disease with hr-HPV infection that rapidly (<5ys) progressed to AIN-HG and anal SCC. Finally, osseous metastases occurred and the patient died shortly after. Conclusions: This case of a patient diagnosed with SCC of the anal canal in combination with Crohn's disease as well as HPV Type 16 infection, points to the pathomechanism leading to dysplasia and finally cancer. We assume that immunosuppressive therapy in Crohn's disease may predispose to both persistent HPV infection and HPV related invasive anal carcinoma. The accelerated progression of HPV associated neoplasia in immunosuppressed patients might represent a fast-tracked process of the long-term course of precancerous or cancerous lesions in immunocompetent hosts. This might implicate, that there is a need to re-evaluate current screening guidelines for anal cancer in patients with chronic inflammatory bowel disease under immunosuppressive therapy.
\end{abstract}

Keywords: Crohn's disease; Human papillomavirus (HPV); anal squamous cell carcinoma; Inflammatory bowel disease (IBD); immunosuppression

\section{Introduction}

Squamous cell carcinoma (SCC) is the most common anal cancerous lesion, most frequently associated with high-risk (hr) HPV infection, especially in women. The prevalence of hr HPV type (high risk HPV type) $16 / 18$ is approximately $70 \%$ in invasive and in-situ SCC (AIN “high grade").

Further, anal cancer has a sexually transmitted etiology and in this context HPV Type 16 is found in the majority of anal cancer lesions [1, 2]. There is evidence that Crohn's disease predisposes to adenocarcinoma in patients with perianal disease $[3,4]$. In context with inflammatory bowel disease (IBD), the risk of cancer is predominantly associated with duration and severity [5]. Usually it takes more than 15 years to progress from chronic inflammation caused by IBD to cancer [6].
Expression of the tumor suppressor p16 $6^{\text {INk4a }}(p 16)$ has been proposed as a surrogate marker for transforming hr-HPV infection. It's overexpression is thought to reflect

*Corresponding author: Anna Fischer, Institute of Pathology Nordhessen, Germaniastraße 7-9, 34119 Kassel, Germany. Tel.: +49-561-50042-108; Fax: +49-561-50042-200; Email: fischer@patho-nordhessen.de

Received 25 June 2016 Revised 16 August 2016 Accepted 12 September 2016 Published 23 September 2016

Citation: Fischer AK, Krause T, Steinbrück H, Schildhaus HU, Hoppenau C, Hesterberg R, Rüschoff J. Rapid tumor progression in a patient with HPV type 16 associated anal squamous cell carcinoma suffering from longstanding Crohn's disease: A case report. J Cancer Res Ther. 2016; 4(5):50-54. DOI: 10.14312/2052-4994.2016-9

Copyright: (c) 2016 Fischer AK, et al. Published by NobleResearch Publishers. This is an open-access article distributed under the terms of the Creative Commons Attribution License, which permits unrestricted use, distribution and reproduction in any medium, provided the original author and source are credited. 
the presence of biologically active HPV infection, due to the fact that functional inactivation of $p R b$ by viral E7 induces p16 ${ }^{\mathrm{INK} 4 \mathrm{a}}$ upregulation [7]. From a biological point of view p16/Ki67 dual-stain within the same cell (nucleus) is regarded as a hallmark of cell-cycle deregulation. In normal cells the expression of p16 and Ki67 is mutually exclusive [8].

Herein we present a patient with long-standing fistulating Crohn's disease ( $\sim 30$ years) who went through several surgeries including ileocolectomy and multiple anal fistula resections, combined with immunosuppressive therapy additionally periodically since 2008. In 2014, he was diagnosed with HPV high risk associated metastasized anal cancer and died almost one year later.

Tumorbiological, diagnostic and clinical significance of HPV infection in long-standing Crohn's disease/IBD is demonstrated.

\section{Materials and methods}

In total nine tissue samples ( 6 biopsies and 3 resection specimens) were diagnosed at the Institute of Pathology Nordhessen obtained from 1988 through 2014 in a 57years old patient with long-standing history of Crohn's disease, first radically treated in 1985 by ileocolectomy.

Specimens were routinely stained by $\mathrm{H} \& \mathrm{E}$ and immunohistochemistry (IHC) was performed on nearly all formalin-fixed paraffin embedded tissues using Ventana automated Immunostainer system (Ventana Medical Systems, Tucson, AZ, USA).

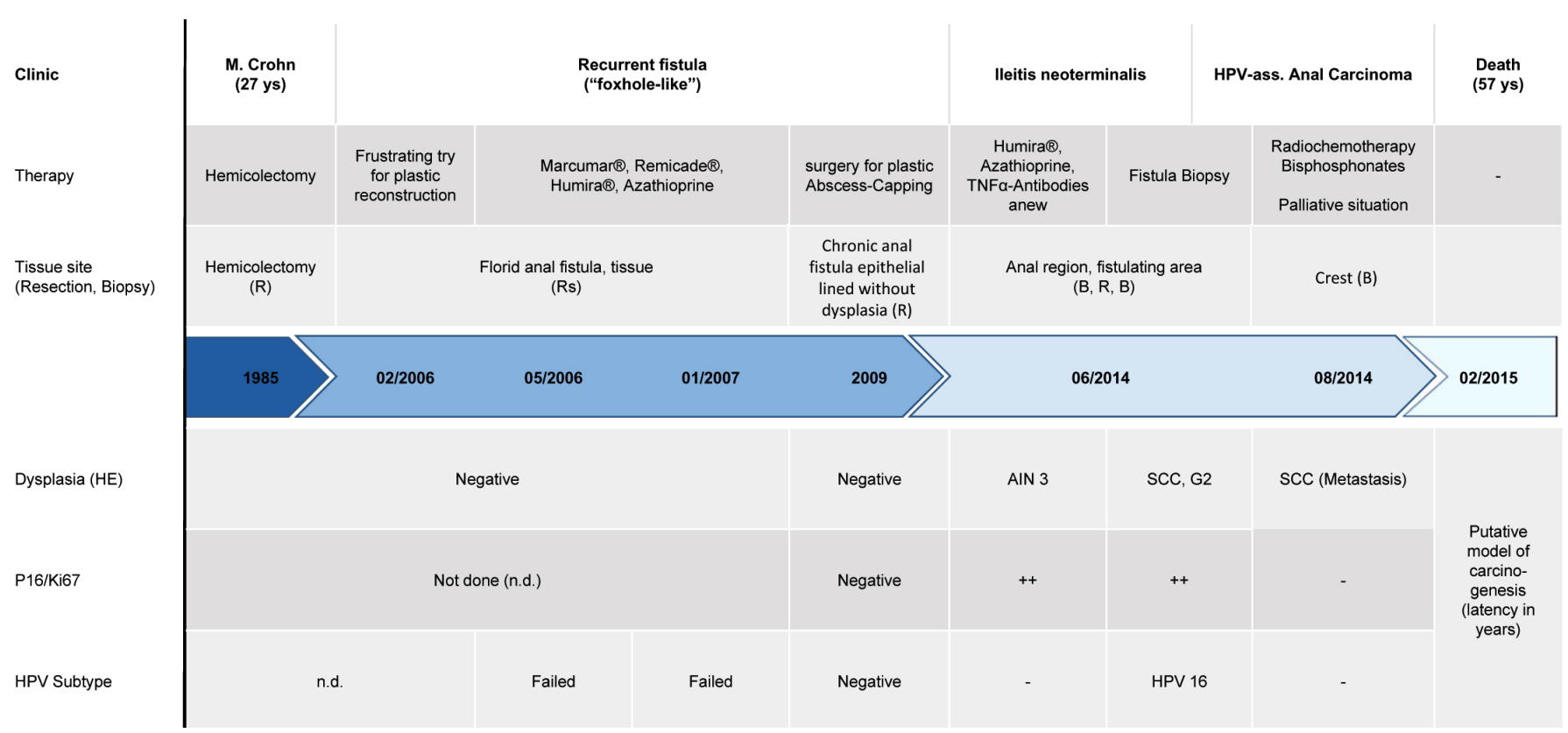

Figure 1 Patients' history, Chronic.

Additionally, the patient received immunosuppressive treatment (Infliximab $\triangleq$ Remicade ${ }^{\circledR}, \quad$ Adalimumab $\triangleq$ Humira ${ }^{\circledR}$, Azathioprine, Mesalazine $\triangleq$ Salofalk ${ }^{\circledR}$, Prednisolon) which could ease the pain and lead to nearly complete restitution of fistulas. After stopping the drug therapy, the patient immediately relapsed.
For immunostaining PanCK (mouse anti-Cytokeratin broad spectrum (AE1 \& AE3) antibody, dilution 1:100, by Zytomed Systems $\left.{ }^{\circledR}\right)$, p16 (CINtec ${ }^{\circledR}$ p16 with monoclonal mouse anti-p16 ${ }^{\mathrm{INK} 4 \mathrm{a}}$ (E6H4, ready to use primary antibody)) and Ki67 (rabbit anti-human Ki67 polyclonal antibody, dilution 1:200, by Spring Bioscience ${ }^{\circledR}$ ) were used. For p16/ Ki67 double staining procedure, the same antibodies and dilutions as for monostaining were used. P16-antibody was applied first, followed by UltraView Universal detection kit, with Diaminobenzidine and Horseradish Peroxidase. Then the secondary antibody (Ki67) was applied, followed by an UltraView Red universal detection kit (Alkaline Phosphatase Red Detection Kit, Roche, biotin-free), marking all Ki67positive cells red. Evaluation of p16/Ki67 IHC double staining was focused on co-expressing tumor cells.

HPV subtyping (done at Institute of Pathology University of Göttingen) by F-HPV typing ${ }^{\mathrm{TM}}$ using a multiplex PCR with fragmentation length analysis on an ABI 3500 genetic analyzer. Extracted DNA was amplified using the analyzer with a set of 16 fluorescently labelled primers, detecting HPV types, especially high-risk types $(6,11,16,18,31,33$, $35,39,45,51,52,56,58,59,68)$ as well as a human STR (short tandem repeats) for internal control. On samples older than 2009, it was impossible to gain exploitable material for molecular HPV subtyping.

\section{Clinical history and findings}

A HIV-negative (human immunodeficiency virus) patient born in 1957 underwent hemicolectomy and multiple anal fistula resection surgeries thereafter, because of Crohn's disease and ongoing tissue damage (1985 till 2014). All specimens were examined by our team (Figure 1). 
evidence of already invasive growth as G2 non-keratinizing SCC (Figure 2c), histologically. Whereas HPV subtyping was impossible due to sample conditions in the first fistula resection specimen in 2006. P16 immunostaining was not performed.

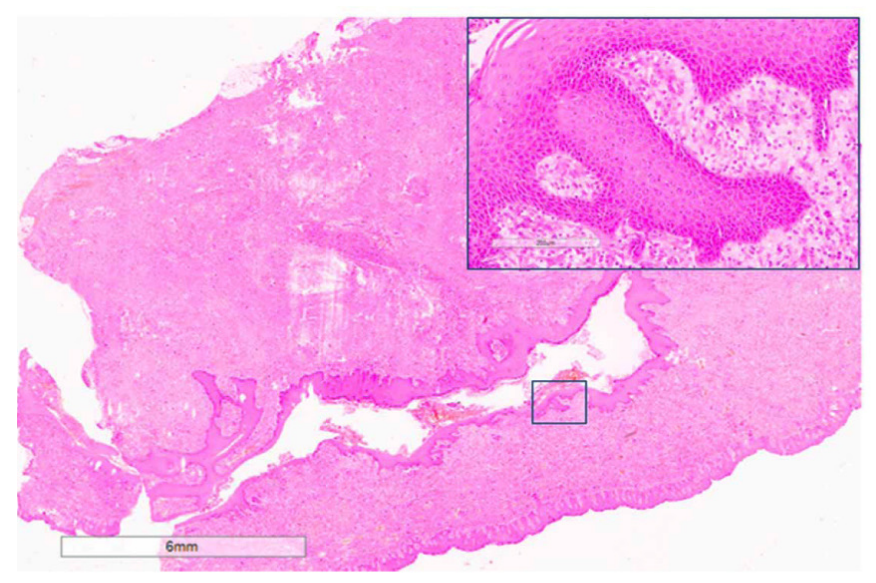

(a)

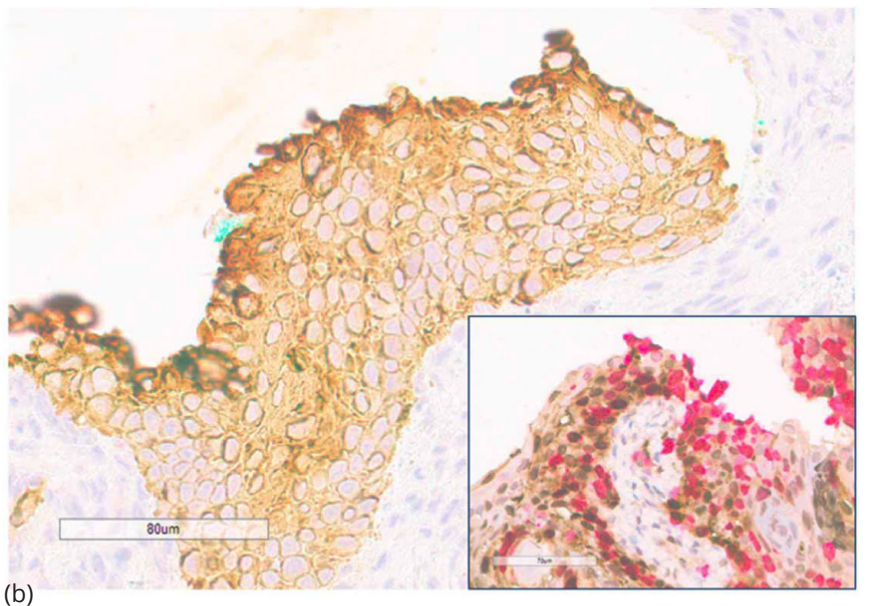

(b)
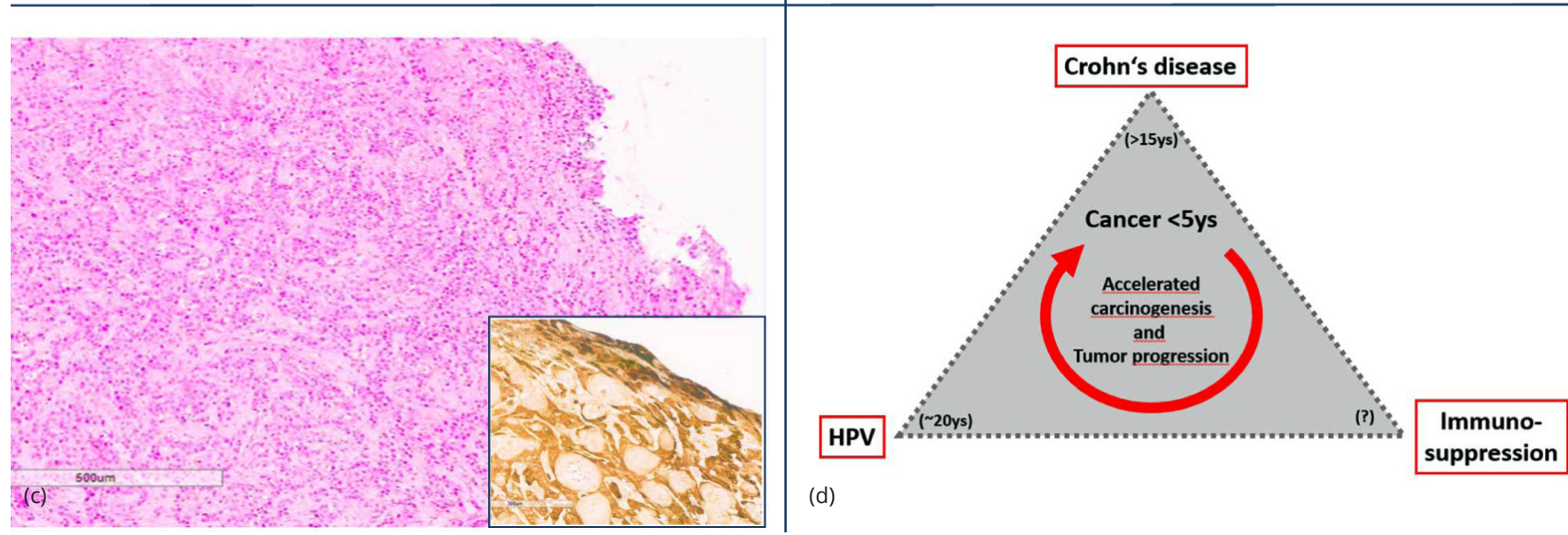

Figure 2 Histological and immunhistological findings: (a) Anal resection specimen showing a longitudinally sectioned fistula covered by non-keratinisized squamous epithelium (H\&E, 2.5x) with elongated rete ridges but no evidence of dysplasia (Insert: H\&E, 20x); (b) Fistula biopsy demonstrating high grade anal in-situ neoplasia (AIN) with no infiltrative growth (Pan CK, 5x), increased Ki67 proliferative activity and strong p16 positivity with some nuclei showing co-expression of both markers (Insert: p16/Ki67 double staining, 40x); (c) Anal fistula biopsy presenting an invasive anal squamous cell carcinoma (H\&E, 10x) strongly immunostained by p16 (Insert: 20x); (d) Pathogenetic model of rapid carcinogenesis in Crohn's disease due to immunosuppressive therapy and hrHPV infection.

Excisions taken in 2007 and 2009 turned out to be negative by p16/Ki67 double-staining immunohistochemistry. Retrospective HPV PCR based analysis in 2009-obtained tissue did not show any detectable HPV DNA.

In contrast, the AIN-HG and invasive SCC samples turned out to be strongly positive for p16/Ki67 staining and molecular analysis disclosed a HPV-16 high-risk infection.

Finally, the patient's status was defined as Crohn's disease associated with high risk HPV infection, rapidly $(<5 y$ ) progressing to AIN-HG and anal SCC, having finally spread with osseous metastases.

In September 2014, this case was presented in an interdisciplinary tumor board, reporting the HPV associated anal carcinoma and multifocal bone metastases in a bone scintigraphy. Biopsy samples from thoracic vertebra segment 9 and iliac crest disclosed a moderately differentiated squamous cell carcinoma which defines the tumor as already having spread by haematogenous metastases. Accordingly, systemic palliative chemotherapy including Bisphosphonates was initiated as well as radiation of the anal tumor and the metastasis in the thoracic vertebra for local control. However, the patient's clinical situation deteriorated and finally he died within palliative care in February 2015.

\section{Discussion}

This case of a patient diagnosed with SCC of the anal canal in combination with both, Crohn's disease as well as HPV Type 16 infection, raises the question about the foremost pathomechanism leading to dysplasia and finally cancer, supporting the dysplasia-carcinoma sequence theory. There have been several case reports suggesting that longstanding Crohn's disease with a history of multiple fistulae and recurrent surgical resections is a risk factor for both adeno- and SCC $[9,10]$. In men, neoplasia of the anoderm is up to four times more frequent than in women, whereas women more often develop lesions in the transitional zone [11]. With respect to the origin of anal SCC there are theories about two main hotspots the neoplasia arises from, namely perianal or within anal mucosa/anal canal. Hr-HPV infection is much more common in intra- 
anal located tumors, whereas neoplasia of the perianal region is less often hr-HPV-positive (92\% vs. 64\%) [11]. In the presented case, the histological investigations were performed on fistula resection specimen from perianal region with extent to depth. Directly intra-anal probe excisions have not been investigated. Clinically, SCC in the anal/perianal region is regarded as perianal if the tumor bulk is visible by inspection distally to the anal verge [12]. Histologically, these tumors typically are of moderate to well differentiated keratinizing type. In contrast, tumors arising from the anal epithelium show mostly a non-keratinizing usually basaloid differentiation. This rather characterizes our tumor as originating from anal mucosa having been irritated by chronic inflammation and fistulization $[11,13]$.

Crohn's disease is a chronic inflammatory bowel disease of gastrointestinal tract. It affects $15-20$ out of 100.000 inhabitants per year and incidence is maximal between the ages of 20 to $30[14,15]$. Its' cause is widely unknown but there are approaches and suggestions for genetic componentsand someenvironmentalfactors. The diagnosis of Crohn's disease is often made by clinical, histological and morphological data. Common complications are fistulas, anal bleedings, abscesses and free perforation with peritonitis [16]. The involvement of the terminal ileum and fistulae are characteristics of Crohn's disease [17]. If perforation occurs, surgical bowel resection according to the general condition of the patient is standard treatment. The risk of intestinal cancer in IBD has been identified in several studies $[5,18]$, so that there is evidence that anal inflammation predisposes to anal cancer [6], both adenoas well as squamous cell carcinoma [9]. The current ECCOguidelines mention anal SCC as a rare complication of IBD (perhaps more common in patients with chronic fistulizing Crohn's disease) which may possibly be associated with carcinogenic HPV types [19].

Hr-HPV infection is a well-established causative factor inducing anal cancer in general [10]. In gynecologic tissue, for example, cervical cancer arises usually within 20 years after infection [20]. In high risk groups, such as HIV infected patients, progression to invasive anal cancer has recently been shown to be very low but increasing $[2,21]$. In general, anal cancer is rare, about 2 cases per 100.000 $[17,10]$ and in the majority of cases it is associated with HPV-HR infection [3, 22-24]. The fact that anal cancer has a sexually transmitted etiology and shows HPV type 16 mono-infection in $65 \%$ to $75 \%$ of the cases this states correlation between cause and effect $[1,2]$.

Like in the cervix, HPV type 16 is predominantly associated with squamous cell carcinoma, whereas type 18 is rather linked with adenocarcinoma. Thereby HPV type 16 monoinfection is also mostly found in cervical cancer samples [25-27].

Our presented patient with long-standing Crohn's disease must have had acquired HPV-HR infection within the past five years before cancer diagnosis as we did not see any dysplasia in several preceding fistula resections. Thus, this case is indicative of an unusual rapid progression from HPV infection to cancer.
Since even in high risk groups such as HIV infected patients progression to invasive anal cancer has recently been stated to be very rare $[21,28]$, it is shown that besides Crohn's disease another compelling factor should be taken into consideration namely the long period of immunosuppressive therapy in this presented patient. In several studies, the impact of immunosuppressive therapy such as azathioprine and corticosteroids in IBD patients has been proven and it is stated that immune-modulator therapy increases the risk for persistent HPV infection [19], additionally to the already increased risk caused by the immunosuppression by the disease itself.

We therefore assume that Crohn's disease along with guideline adjusted treatment may predispose for both persistent HPV-HR infection and rapid transformation into AIN and invasive cancer. This might implicate, that there is need to re-evaluate current screening guidelines for anal cancer in patients with chronic IBD [29], because it is a rare complication but approachable for further screening methods, particularly in men.

As a possible screening method for anal dysplasia, especially for high-risk patients like HIV infected or profoundly immunosuppressively treated ones could be HPV-screens, e.g. once a year or every 2-3 years followed by cytological check-ups, besides digital anal-rectal examination (DARE). HPV16-detection is in $92 \%$ specific for an AIN-HG. In case of highly suspicious findings or positive results, a highresolution anoscopy along with taking a directed biopsy is required, for confirmation of diagnosis and identification of the degree of dysplasia $[30,31]$. However, data for AIN screening effect on incidence of anal carcinoma in patients with Crohn's disease are missing.

Focusing on a few patients suffering from IBD alone, without viral infection, and ending up with anal SCC the mean time was 15-16 years after onset of IBD symptoms but at least more than ten years $[6,10]$. That goes along with current suggestions that anal SCC arises in chronic fistulas following a dysplasia-carcinoma sequence [32]. Research protocols state that KRAS and BRAF mutations in anal carcinoma are rare findings and have no significant impact on overall or disease-free survival [33]. Thus K-Ras mutations have not been looked for in this special case.

Based on our case, we assume that Crohn's disease may predispose for both, persistent hr-HPV infection and potentially to rapid transformation into AIN and invasive cancer, in particular if immunosuppressive therapy is administered. It has also been described by a review from Slesser et al. who stated that in Crohn's disease anal squamous carcinoma presents at younger age with poorer outcome compared to general population [10].

\section{Conclusion}

The presented case indicates rapid oncogenesis ( $<5$ years) after HPV high risk infection in long-standing fistulating Crohn's disease. Thereby, immunosuppressive therapy predisposes for persistent HPV infection and progression to AIN [10,34](Figure2d). For IBD patients routinely endoscopic examinations are already standard of care and it is known that patients benefit from this investigation. Current 
recommendations for HPV-vaccination in IBD patients exist. Vaccines are best given before immunomodulator therapy, explicitly including HPV. Though, perianal fistula in combination with immunomodulatory therapy - as most IBD patients receive - may provide a target point for HPV viruses [10]. Since anal squamous carcinoma is a rare entity, focused screening for HPV in IBD patients, especially in men, e.g. by anal smears or probe excisions from chronic fistulizing areas, are currently not a routine diagnostic standard and should perhaps be considered for screening recommendations.

\section{Acknowledgements}

This study was supported by the staff within the laboratory of H-U. Schildhaus and C. Hoppenau who did the HPV subtyping at the University Pathology Institute of Göttingen. Further, R. Hesterberg and H. Steinbrück performed surgical examination and sample recovery, and T. Krause cared about routine medical surveillance and screening as well as follow-up investigations for the patient. I gratefully acknowledge U. Hampacher and her team, who did immunohistochemistry within the laboratory of the Institute of Pathology Nordhessen.

\section{Compliance with ethical standards}

There are no potential conflicts of interest expected. Due to the fact that we present an anonymous clinical case, there should no informed consent be needed.

\section{Funding}

The authors declare that there has been no funding on this project. The used tissue samples were gained by surgical exstirpation and prepared for diagnostics. Followingly, the involved pathologists of the Institute of Pathology Nordhessen collected all existing patient relevant data and specimen for further scientific work.

\section{Conflicts of interest}

The authors declare no conflicts of interest.

\section{Abbreviations}

AIN-HG: anal intraepithelial neoplasia high grade; DARE: digital anal rectal examination; ECCO: European Crohn's and Colitis Organization; HIV: human immunodeficiency virus; HPV-HR: high risk human papilloma virus; IBD: Inflammatory bowel disease; PCR: polymerase chain reaction; SCC: squamous cell carcinoma

\section{References}

[1] Deans GT, McAleer J], Spence RA. Malignant anal Tumours. Br J Surg. 1994; 81(4):500-508.

[2] Darragh TM, Winkler B. Anal cancer and cervical cancer screening: key differences. Cancer Cytopathol. 2011; 119(1):5-19.

[3] Frisch M, Glimelius B, van den Brule AJ, Wohlfahrt J, Meijer CJ, et al. Benign anal lesions, inflammatory bowel disease and risk for highrisk human papillomavirus-positive and-negative anal carcinoma. $\mathrm{Br}$ J Cancer. 1998; 78(11):1534-1538.

[4] Hoots BE, Palefsky JM, Pimenta JM, Smith JS. Human papillomavirus type distribution in anal cancer and anal intraepithelial lesions. Int J Cancer. 2009; 124(10):2375-2383.

[5] Eaden JA, Abrams KR, Mayberry JF. The risk of colorectal cancer in ulcerative colitis: a meta-analysis. Gut. 2001; 48(4):526-535.

[6] Frisch $M$, Johansen $C$. Anal carcinoma in inflammatory bowel disease. BrJ Cancer. 2000; 83(1):89-90.

[7] Deng Z, Hasegawa M, Aoki K, Matayoshi S, Kiyuna A, et al. A comprehensive evaluation of human papillomavirus positive status and p16INK4a overexpression as a prognostic biomarker in head and neck squamous cell carcinoma. Int J Oncol. 2014; 45(1):67-76.

[8] Ikenberg H, Bergeron C, Schmidt D, Griesser H, Alameda F, Angeloni C, et al. Screening for cervical cancer precursors with p16/Ki67 dualstained cytology: Results of the PALMS Study. J Natl Canc Inst. 2013; 105(20):1550-1557.

[9] Riddell R, Jain D. Lewin Gastrointestinal pathology and its clinical implications. Wolters Kluwer. 2nd edition. 2014.

[10] Slesser AA, Bhangu A, Bower M, Goldin R, Tekkis PP. A systematic review of anal squamous cell carcinoma in inflammatory bowel disease. Surgical Oncol. 2013; 22(4):230-237.

[11] Stolte, Manfred, Rüschoff, Josef, Klöppel, et al. Pathologie. Verdauungstrakt und Peritoneum. 2013; 708-721.

[12] Bosman FT, Carneiro F, Hruban RH, Theise ND. WHO Classification of Tumours of the digestive System. World Health Organization Classification of Tumours. IARC. 4th Edition. 2010; Vol 3.

[13] Leonard D, Beddy D, Dozois EJ. Neoplasms of the Anal Canal and Perianal Skin. Clin Colon Rectal Surg. 2011; 24(1):54-63.

[14] Duchmann R. Chronisch entzündliche Darmerkrankungen. Hess Aerzteblatt. 2014; 690-697.

[15] Loftus EV. Clinical epidemiology of inflammatory bowel disease: Incidence, prevalence and environmental influence. Gastroenterology. 2004; 126(6):1504-1517.

[16] Schwartz DA, Pemberton JH, Sandborn WJ. Diagnosis and treatment of perianal fistulas in Crohn disease. Ann Intern Med. 2001; 135(10):906918.

[17] Wagner M, Lefevre JH, Royer B, Svrcek M, Pradel C, et al. Internal fistula leakage due to a road traffic accident: a fortuitous diagnosis of Crohn's disease. J Crohns Colitis. 2012; 6(5):603-605.

[18] Jess T, Loftus EV, Velayos FS, Harmsen WS, Zinsmeister AR, et al. Risk of intestinal cancer in inflammatory bowel disease: A population-based study from olmsted County, Minnesota. Gastroenterology. 2006; 130(4):1039-1046.

[19] Rahier JF, Magro F, Abreu C, Armuzzi A, Ben-Horin S, et al. Second European evidence-based consensus on the prevention, diagnosis and management of opportunistic infections in inflammatory bowel disease. J Crohns Colitis. 2014; 8(6):443-468.

[20] McIndoe WA, McLean MR, Jones RW, Mullins PR. The invasive potential of carcinoma in situ of the cervix. Obstet Gynecol. 1984; 64(4):451458.

[21] Mathews WC, Agmas W, Cachay ER, Cosman BC, Jackson C. Natural history of anal dysplasia in an HIV-infected clinical care cohort: estimates using multi-state Markov modeling. PLoS One. 2014; 9(8):e104116.

[22] Roldán Urgoiti GB, Gustafson K, Klimowicz AC, Petrillo SK, Magliocco $\mathrm{AM}$, et al. The prognostic value of HPV status and p16 expression in patients with carcinoma of the anal canal. PLOS One. 2014; 9(10):e108790.

[23] Chaturvedi AK. Beyond cervical cancer: burden of other HPV-related cancers among men and women. J Adolesc Health. 2010; 46(4 suppl):S20-S26.

[24] Abramowitz L, Jacquard AC, Jaroud F, Haesebaert J, Siproudhis L, et al. Human papillomavirus genotype distribution in anal cancer in France: The EDiTH V study. Int J Cancer. 2011; 129(2):433-439.

[25] Cid-Arregui A. Therapeutic Vaccines against human papillomavirus and cervical cancer. Open Virol J. 2009; 3:67-83.

[26] He J, Ji Y, Li A, Zhang Q, Song W, et al. MiR-122 directly inhibits human papillomavirus E6 Gene and enhances Interferon signaling through blocking suppressor of Cytokine signaling 1 in $\mathrm{SiHa}$ Cells. PLOS One. 2014; 9(9):e108410.

[27] Rödel F, Wieland U, Fraunholz I, Kitz J, Rave-Fränk M, et al. Human papillomavirus DNA load and p16INK4a expression predict for local control in patients with anal squamous cell carcinoma treated with chemotherapy. Int J Cancer. 2015; 136(2):278-288.

[28] Collett D, Mumford L, Banner NR, Neuberger J, Watson C. Comparison of the incidence of malignancy in recipients of different types of organ: A UK Registry audit. Am J Transplant. 2010; 10(8):1889-1896.

[29] Egan L, D'Inca R, Jess T, Pellino G, Carbonnel F, et al. Non-colorectal intestinal tract carcinomas in inflammatory bowel disease: results of the 3rd ECCO Pathogenesis Scientific Workshop (II). J Crohns Colitis. 2014; 8(1):19-30.

[30] AWMF Condylomata acuminata und andere HPV-assoziierte Krankheitsbilder von Genitale, Anus und Harnröhre. S1-Leitlinie.

[31] Anal dysplasia and anal cancer in HIV-infected: prevention, diagnosis and treatment. S2k-Leitlinie. 2013.

[32] Slater G, Greenstein A, Aufses AH. Anal carcinoma in patients with Crohn's disease. Ann Surg. 1984; 199(3):348-350.

[33] Serup-Hansen E, Linnemann D, Høgdall E, Geertsen PF, Havsteen H. KRAS and BRAF mutations in anal carcinoma. APMIS. 2015; 123(1):5359.

[34] Patel HS, Silver AR, Levine T, Williams G, Northover JM. Human papillomavirus infection and anal dysplasia in renal transplant recipients. Br J Surg. 2010; 97(11):1716-1721. 